\title{
Correction to: "Shelf" Technique Using a Novel Braided Self-Expandable Stent for the Treatment of Wide-Necked Bifurcation Aneurysms
}

\author{
Volker Maus $^{1}(\mathbb{D}) \cdot$ Werner Weber $^{1} \cdot$ Sebastian Fischer ${ }^{1}$
}

Published online: 28 September 2021

(c) The Author(s) 2021

\section{Correction to:}

\section{Clin Neuroradiol 2021}

https://doi.org/10.1007/s00062-021-01032-2

The original version of this article unfortunately contained a mistake. The presentation of Table 1 was incorrect. The corrected Table 1 is given below.

The original article has been corrected.

Open Access This article is licensed under a Creative Commons Attribution 4.0 International License, which permits use, sharing, adaptation, distribution and reproduction in any medium or format, as long as you give appropriate credit to the original author(s) and the source, provide a link to the Creative Commons licence, and indicate if changes were made. The images or other third party material in this article are included in the article's Creative Commons licence, unless indicated otherwise in a credit line to the material. If material is not included in the article's Creative Commons licence and your intended use is not permitted by statutory regulation or exceeds the permitted use, you will need to obtain permission directly from the copyright holder. To view a copy of this licence, visit http://creativecommons.org/licenses/by/4. $0 /$.

The online version of the original article can be found under https://doi.org/10.1007/s00062-021-01032-2

Volker Maus

volker.maus@kk-bochum.de

1 Department of Diagnostic and Interventional Neuroradiology and Nuclear Medicine, Universitätsklinikum Knappschaftskrankenhaus Bochum, Universitätsklinik der Ruhr-Universität Bochum, In der Schornau 23-25, 44892 Bochum, Germany 
Table 1 Individual overview of patients treated with LVIS EVO "shelf" technique

\begin{tabular}{|c|c|c|c|c|c|c|c|c|c|c|c|}
\hline No. & $\begin{array}{l}\text { Age } \\
\text { (years)/sex }\end{array}$ & Site & $\begin{array}{l}\text { Dome } \\
{[\mathrm{mm}] /} \\
\text { D/N-Ratio }\end{array}$ & $\begin{array}{l}\text { Alpha } \\
\text { angle }^{\text {b }}\end{array}$ & $\begin{array}{l}\text { Previous } \\
\text { Treatment }\end{array}$ & $\begin{array}{l}\text { Stent } \\
\text { Size }\end{array}$ & $\begin{array}{l}\text { Immediate } \\
\text { RROC }\end{array}$ & $\begin{array}{l}\text { Intraprocedural } \\
\text { complication }\end{array}$ & $\begin{array}{l}\text { DSA } \\
\text { FU } \\
\text { (days) }\end{array}$ & $\begin{array}{l}\text { FU } \\
\text { RROC }\end{array}$ & $\begin{array}{l}\text { FU } \\
\text { mRS }\end{array}$ \\
\hline 1 & $47 / F$ & MCA & $2.9 / 1.4$ & 19 & $\mathrm{~N}$ & $3 \times 28$ & 1 & $\mathrm{~N}$ & $\mathrm{~N} / \mathrm{A}^{\mathrm{a}}$ & $\mathrm{N} / \mathrm{A}^{\mathrm{a}}$ & 0 \\
\hline 2 & $59 / \mathrm{M}$ & MCA & $3.5 / 1$ & 24 & $\mathrm{~N}$ & $3 \times 24$ & 1 & $\mathrm{~N}$ & $\mathrm{~N} / \mathrm{A}^{\mathrm{a}}$ & $\mathrm{N} / \mathrm{A}^{\mathrm{a}}$ & 0 \\
\hline 3 & $58 / \mathrm{F}$ & MCA & $4.0 / 0.9$ & 9 & $\mathrm{~N}$ & $3 \times 24$ & 2 & $\mathrm{~N}$ & 96 & 2 & 0 \\
\hline 4 & $61 / \mathrm{F}$ & PcaA & $1.8 / 1.2$ & 24 & Coiling & $2.5 \times 22$ & 1 & $\mathrm{~N}$ & 96 & 1 & 0 \\
\hline 5 & $61 / \mathrm{M}$ & AcomA & $1.3 / 1.0$ & 4 & $\mathrm{~N}$ & $2.5 \times 22$ & 1 & $\mathrm{~N}$ & $\mathrm{~N} / \mathrm{A}^{\mathrm{a}}$ & $\mathrm{N} / \mathrm{A}^{\mathrm{a}}$ & 0 \\
\hline 6 & $56 / \mathrm{M}$ & $\mathrm{MCA}$ & $1.5 / 1.5$ & 26 & Coiling & $2.5 \times 22$ & 1 & $\mathrm{~N}$ & 115 & 1 & 0 \\
\hline 7 & $56 / \mathrm{M}$ & MCA & $3.1 / 0.9$ & 17 & $\mathrm{~N}$ & $3 \times 24$ & 1 & $\mathrm{~N}$ & 133 & 1 & 0 \\
\hline 8 & $50 / \mathrm{F}$ & ICA-T & $3.2 / 1.6$ & 47 & $\mathrm{~N}$ & $3.5 \times 22$ & 1 & $\mathrm{~N}$ & 193 & 1 & 0 \\
\hline 9 & $38 / \mathrm{F}$ & AcomA & $5.6 / 1.1$ & 72 & Coiling & $2.5 \times 22$ & 1 & $\mathrm{~N}$ & 89 & 1 & 0 \\
\hline 10 & $52 / \mathrm{F}$ & MCA & $1.9 / 1.7$ & 29 & $\mathrm{~N}$ & $2.5 \times 17$ & 1 & $\mathrm{~N}$ & 98 & 1 & 0 \\
\hline 11 & $65 / \mathrm{M}$ & AcomA & $4.7 / 1.2$ & 38 & $\mathrm{~N}$ & $2.5 \times 27$ & 1 & $\mathrm{~N}$ & 7 & 1 & 1 \\
\hline 12 & $54 / \mathrm{M}$ & $\mathrm{MCA}$ & $1.6 / 0.7$ & 27 & $\mathrm{~N}$ & $2.5 \times 17$ & 1 & $\mathrm{~N}$ & 357 & 1 & 0 \\
\hline 13 & $48 / \mathrm{F}$ & $\mathrm{MCA}$ & $1.3 / 1.5$ & 38 & Coiling & $3 \times 24$ & 1 & $\mathrm{~N}$ & 399 & 1 & 0 \\
\hline 14 & $62 / F$ & AcomA & $2.0 / 1.0$ & 43 & $\mathrm{~N}$ & $3 \times 24$ & 1 & $\mathrm{~N}$ & 419 & 1 & 0 \\
\hline 15 & $67 / \mathrm{M}$ & MCA & $4.7 / 1.3$ & 11 & $\mathrm{~N}$ & $3 \times 28$ & 1 & $\mathrm{~N}$ & $\mathrm{~N}^{\prime} \mathrm{A}^{\mathrm{a}}$ & $\mathrm{N}^{-\mathrm{A}^{\mathrm{a}}}$ & 0 \\
\hline
\end{tabular}

AcomA anterior communicating artery, $D / N$ dome-to-neck, $D S A$ digital subtraction angiography, $F U$ follow-up, $M C A$ middle cerebral artery, $m R S$ modified Rankin scale, N/A not available, PcaA pericallosal artery, RROC Raymond-Roy occlusion classification, $N$ no, $M$ male, $F$ female aStill pending at the time of submission

${ }^{\mathrm{b}}$ Angle between aneurysm and parent artery 